\title{
Técnica de reconstrucción en roturas recidivantes del tendón patelar
}

\section{Reconstruction Technique in Recurrent Patellar Tendon Ruptures}

\author{
Pedro Díaz Allende ${ }^{1}$ Andrés Cárdenas López ${ }^{2}$ Alexander Pérez Acevedo ${ }^{1}$ Joel Hernández De León ${ }^{1}$ \\ Julio Hernández Echazabal ${ }^{1}$ \\ ${ }^{1}$ Servicio de Ortopedia y Traumatología, Hospital Ricardo Valenzuela \\ Sáez, Rengo, Chile \\ 2 Médico-Cirujano adjunto, Hospital Ricardo Valenzuela Sáez, Rengo, \\ Chile \\ Address for correspondence Pedro Díaz Allende, MD, Servicio de \\ Ortopedia y Traumatología, Hospital Ricardo Valenzuela Sáez, Av. \\ Renato Correa Labra 210, Rengo, Chile \\ (e-mail: drpedrodiaz@gmail.com).
}

Rev Chil Ortop Traumatol 2017;58:59-65.

\section{Resumen \\ Palabras Clave \\ - recidivante \\ - rotura \\ - técnica reconstrucción \\ - tendón patelar \\ - tendón rotuliano}

La rotura recidivante del tendón patelar es infrecuente y generalmente asociada a comorbilidad sistémica que altera la interfaz tendón-hueso o a consecuencia de un retorno inadecuado a actividades, previo a completar el proceso de rehabilitación. La reconstrucción quirúrgica se basa en el aumento tendíneo con autoinjertos o aloinjertos. El uso de autoinjertos evita el riesgo de transmisión de enfermedades infecciosas y costos en relación a los aloinjertos y no requiere un segundo tiempo quirúrgico para remover material utilizado en la protección del tendón reconstruido, en el caso de utilizar refuerzos con alambres de cerclaje o cables de acero. Se presenta la reconstrucción del tendón patelar con autoinjerto semitendinoso-gracilis, por ser una técnica validada en la literatura, que restablece la fuerza y la estabilidad del mecanismo extensor de forma costo-efectiva y permite el retorno adecuado a las actividades de la vida diaria.

Recurrent rupture of the patellar tendon is infrequent and usually associated with systemic comorbidity that alters the tendon-bone interface or as a result of an inadequate return to activities, prior to completing the rehabilitation process. Surgical reconstruction is based on tendon augmentation with autografts or allografts. The use of autografts avoids the risk of infectious diseases and costs in relation to allografts and does not require a second surgical time to remove material used in the protection of the reconstructed tendon, in case of using cerclage wires or steel cables reinforcements. Reconstruction of the patellar tendon with semitendinosus-gracilis autograft is presented as a technique validated in the literature, which restores the strength and stability of the extensor mechanism in a cost-effective way and allows adequate return to activities of daily living. received

May 6, 2017

accepted

July 28, 2017

published online

August 23, 2017
DOI https://doi.org/

10.1055/s-0037-1606252.

ISSN 0716-4548.
Copyright (e) 2017 by Thieme Revinter

Publicações Ltda, Rio de Janeiro, Brazil
License terms

(요 (1) $\Theta \circledast$ 


\section{Introducción}

Dentro de las lesiones del aparato extensor de rodilla, la rotura del tendón patelar es la menos frecuente, con una incidencia de 0,68/100,000 por año, ${ }^{1}$ siendo 6 veces menos frecuente que la fractura de rótula. ${ }^{2}$ En su presentación aguda, se encuentran involucrados mecanismos indirectos de sobrecarga excéntrica del cuádriceps con rodilla en flexión y pie en carga. Epidemiológicamente, se presentan en pacientes activos menores de 40 años y en su mayoría de sexo masculino, ${ }^{3}$ en relación 4:1. En su etiopatogenia ${ }^{4-8}$ son importantes el microtrauma repetitivo y la tendinosis sintomática no tratada, las que alteran la estructura del colágeno y la microcirculación local. Factores de riesgo conocidos son la utilización de corticoides locales inyectables o sistémicos orales de uso crónico. En lesiones agudas, la rotura se ubica típicamente en la unión osteotendínea proximal, por lo que el tratamiento se basa en su reinserción al polo inferior de la rótula mediante suturas transóseas, con buenos resultados funcionales y tasas de fallo de un $5 \%$ (reparación primaria simple), versus $2 \%$ con refuerzo mediante cerclaje de alambre, cable de acero o suturas de alta resistencia no reabsorbibles. ${ }^{9}$

La cirugía de reconstrucción del tendón patelar por fallo en la reparación primaria (recidiva), son en extremo infrecuentes y su prevalencia es desconocida. Se asocian a pacientes con comorbilidades específicas (endocrinopatías, enfermedades inflamatorias sistémicas, insuficiencia renal crónica), que debilitan de forma crónica la interfaz huesotendón y mecanismos lesionales de baja energía. ${ }^{10}$

Se han propuesto múltiples técnicas de reconstrucción, ${ }^{9}$ donde se destacan: autoinjertos de tendón semitendinoso, ${ }^{11}$ semitendinoso-gracilis (STG), ${ }^{12,13}$ autoinjerto contralateral de hueso-tendón patelar-hueso $(\mathrm{HTH}),{ }^{14,15}$ aloinjerto $\mathrm{HTH}^{16}$ aloinjerto de tendón de Aquiles ${ }^{17-19}$ y de forma excepcional en la literatura materiales sintéticos. ${ }^{20-23}$ Sin embargo, aún es controversial el tratamiento de elección, debido principalmente a su baja incidencia y a la inexistencia de estudios biomecánicos y clínicos que comparen las distintas técnicas.

Se presenta la reconstrucción del tendón patelar con autoinjerto STG, por ser una técnica validada en la literatura, $, 13,24-27$ que restablece la fuerza y estabilidad del mecanismo extensor de forma costo-efectiva y permite el retorno adecuado a las actividades de la vida diaria prelesionales, con tasas muy bajas de complicaciones.

\section{Técnica Quirúrgica}

Con el paciente bajo anestesia general o regional con sedación, se posiciona en decúbito supino con la extremidad inferior afectada en semiflexión. Se realiza un abordaje sobre la pata de ganso ipsilateral y se obtienen los tendones STG con stripper abierto para preservar su inserción tibial, justificado en la preservación de la vascularidad del injerto, evitando fenómenos de necrosis que retrasen su maduración, reducción de la morbilidad del sitio donante y estabilidad adicional en las etapas iniciales de osteointegración. ${ }^{28}$

Posteriormente se realiza un abordaje longitudinal anterior de rodilla, idealmente sobre la cicatriz previa si se trata de

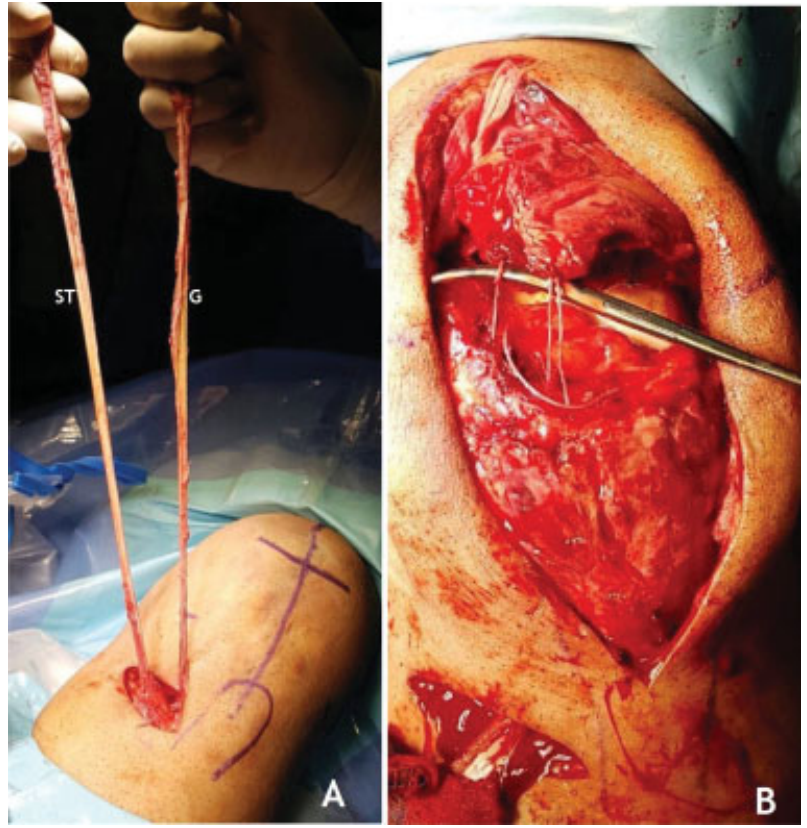

Fig. 1 Abordajes para la cirugía de reconstrucción del tendón patelar. (A) Abordaje sobre la pata de ganso ipsilateral para la cosecha de injertos semitendinoso (ST) y gracilis (G), preservando su inserción tibial utilizando stripper abierto. (B) Abordaje longitudinal anterior de rodilla sobre cicatriz previa. Se evidencia el fallo en la reparación primaria a nivel del polo inferior de la rótula.

una revisión por rerotura. La exposición del aparato extensor debe ser amplia, desde el tendón cuadricipital hasta aproximadamente $3 \mathrm{~cm}$ distal a la tuberosidad anterior de la tibia (TAT), con el fin de realizar una adecuada liberación de adherencias tendíneas ( $\mathbf{- F i g . 1} \mathbf{1}$ ).

Se prueba la longitud de los autoinjertos STG a través del trayecto que recorrerán para la aumentación. Van der Zwaal y $\mathrm{col}^{29}$ y Jarvela y $\operatorname{col}^{30}$ reportan casos utilizando autoinjerto STG libre y fijación ósea con tornillos bioabsorbibles y grapas. Eso es útil ante injertos cortos, que no logren atravesar el túnel horizontal patelar desde su inserción en la pata de ganso, debiéndolos liberar de ésta y reinsertarlos proximalmente.

La mayoría de las lesiones se ubican inmediatamente por debajo del polo inferior de la rótula, en la interfaz osteotendínea. Se debe realizar el desbridamiento local de la fibrosis y estimulación de la medula ósea mediante decorticación del polo inferior de la rótula con cureta, gubia o fresa, para lograr un mejor ambiente biológico para la cicatrización.

A continuación, se planifica un túnel horizontal sobre el tercio medio de la rótula con la ayuda de un compás tibial de reconstrucción ligamentaria, una aguja guía y una broca canulada de $4,5 \mathrm{~mm}$, que posteriormente alojará el paso de los autoinjertos. En ese punto, deben tomarse todas las precauciones para evitar lesionar la superficie articular de la patela. Un segundo túnel óseo de 4,5mm de diámetro se broca de forma oblicua a nivel tibial por sobre la pata de ganso y al menos $2 \mathrm{cms}$ posterior a la TAT, en dirección caudo-cefálica y sentido oblicuo, de medial a lateral, asistido con el compás tibial. Esa configuración evita un killer turn que estrese el injerto en su salida en la porción lateral de la tibia (-Fig. 2). 


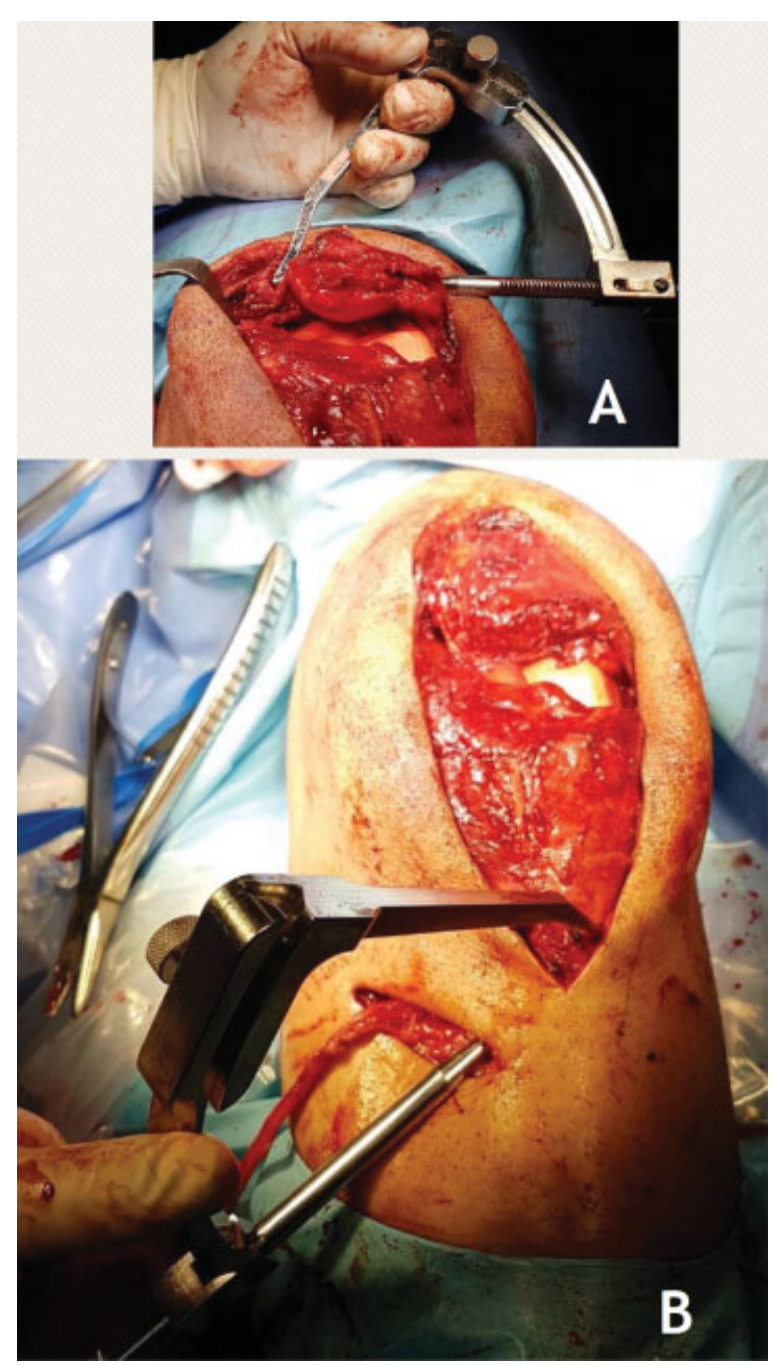

Fig. 2 Preparación de los túneles óseos a nivel patelar (A) y tibial (B) con ayuda de un compás tibial de reconstrucción ligamentaria.

Tras la preparación habitual de los extremos libres de ambos tendones con suturas de alta resistencia $\mathrm{n}^{\circ} 2$, se procede a pasar el injerto semitendinoso, de mayor diámetro y longitud, a través del túnel tibial (de medial a lateral) y luego a través del túnel patelar (de lateral a medial), mediante una aguja guía con ojal (-Fig. 3). El injerto gracilis atraviesa el túnel patelar en sentido contrario (de medial a lateral) desde su inserción nativa. Así, el túnel rotuliano queda completamente cubierto. Se estima que el diámetro combinado de esos injertos (duplicados) varía en rangos que fluctúan desde los 4 a los $6,5 \mathrm{~mm} \cdot{ }^{26}$

En revisiones, se instalan 2 anclas de titanio en el polo inferior de la rótula y se resutura el tendón lesionado con la técnica de Krackow, ${ }^{31}$ para asegurar una adecuada dispersión de las fuerzas a lo largo de la sutura. Las anclas deberían fijarse a aproximadamente $2 \mathrm{~mm}$ de la superficie articular. El colocarlas más superficialmente incrementa las fuerzas de reacción patelofemoral, alterando la biomecánica $\operatorname{articular}^{32}$ (-Fig. 4).

Después de realizar esa configuración rectangular, ambos injertos se tensan y suturan entre sí de forma latero-lateral en

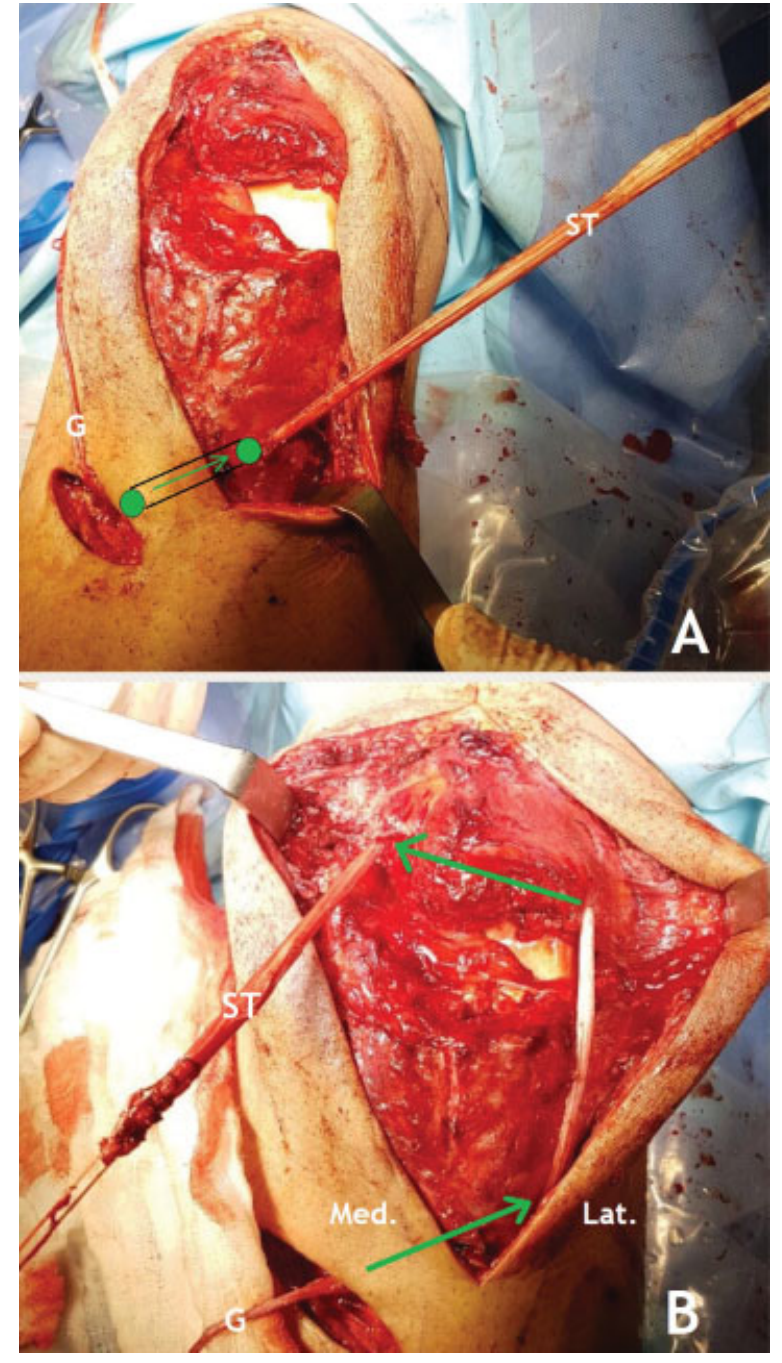

Fig. 3 Trayecto del tendón semitendinoso (ST) a través de los túneles óseos. (A) Paso del ST dentro del túnel tibial (esquematizado en flechas verdes), desde su inserción nativa hacia la inserción lateral. (B) Paso del ST dentro del túnel patelar, de lateral (Lat.) a medial (Med.). Abreviaciones: G, tendón gracilis; Lat, lateral; Med, medial; ST, tendón semitendinoso.

$30^{\circ}$ de flexión de rodilla con sutura irreabsorbible de polipropileno para mejorar la estabilidad del constructo. En esa posición, se asegura que el largo del tendón rotuliano (sin gap lesional visible tras el aumento), sea similar al largo de la patela, preservando así una relación de altura patelar normal según Insall-Savati. ${ }^{33}$ Adicionalmente, se puede incluir la extremidad contralateral en el campo quirúrgico para realizar una comparación de la posición final de la patela.

No existen estudios comparativos respecto al tipo de configuración ideal de los injertos en la reconstrucción, describiéndose la técnica rectangular y en "figura en 8."

El autoinjerto semitendinoso del túnel tibial se fija en $90^{\circ}$ de flexión (evitando así una presión excesiva de la rótula sobre la tróclea femoral), con un tornillo interferencial bioabsorbible de $6 \mathrm{~mm}$ de diámetro para lograr su osteointegración (-Fig. 5). Finalmente, se realiza la reparación de los retináculos con una sutura monofilamento reabsorbile de poliglactina $\mathrm{n}^{\circ} 1 \mathrm{y}$ un punto en cruz. Se inmoviliza la extremidad en extensión 

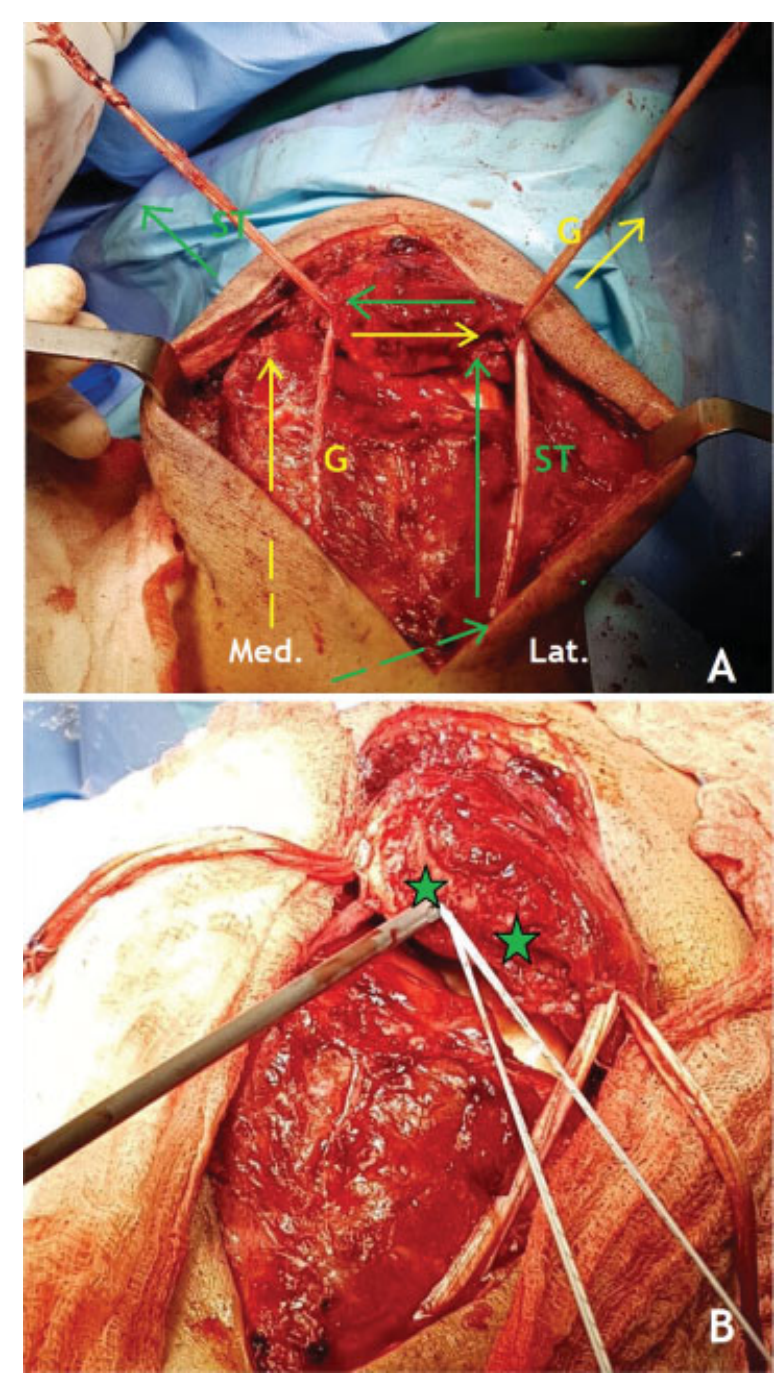

Fig. 4 (A) Configuración de la reconstrucción del tendón patelar con los autoinjertos semitendinoso (ST, en verde) y gracilis ( $\mathrm{G}$, en amarillo). Las flechas indican el trayecto de cada injerto y su dirección. (B) Refuerzo en la reconstrucción mediante anclas al polo inferior de la rótula. Se instalan 2 anclas al polo inferior de la rótula, equidistantes entre sí (las estrellas verdes indican los puntos de inserción). Se realiza la sutura con la técnica de Krackow al tendón patelar.

completa con una órtesis de rodilla con rango articular graduado.

La rehabilitación ${ }^{25}$ comienza al día siguiente con marcha asistida, 2 bastones y carga de peso a tolerancia. Inicialmente, se bloquea la rodilla en extensión completa por 2 semanas. Después se progresa gradualmente, con el objetivo de lograr una movilidad articular entre $0^{\circ}-90^{\circ}$ durante las primeras 6 semanas. Se debe educar desde el comienzo ejercicios isométricos de cuádriceps. Después de 6 semanas, se progresa hacia rangos completos de movilidad y fortalecimiento concéntrico de cuádriceps. Los ejercicios en fase excéntrica se autorizan desde la duodécima semana, trote a partir del sexto mes y retomar actividades deportivas tras 9 meses de rehabilitación, demostrando rangos completos de movilidad articular y al menos un $85 \%$ de la fuerza cuadricipital contralateral en el test isocinético.
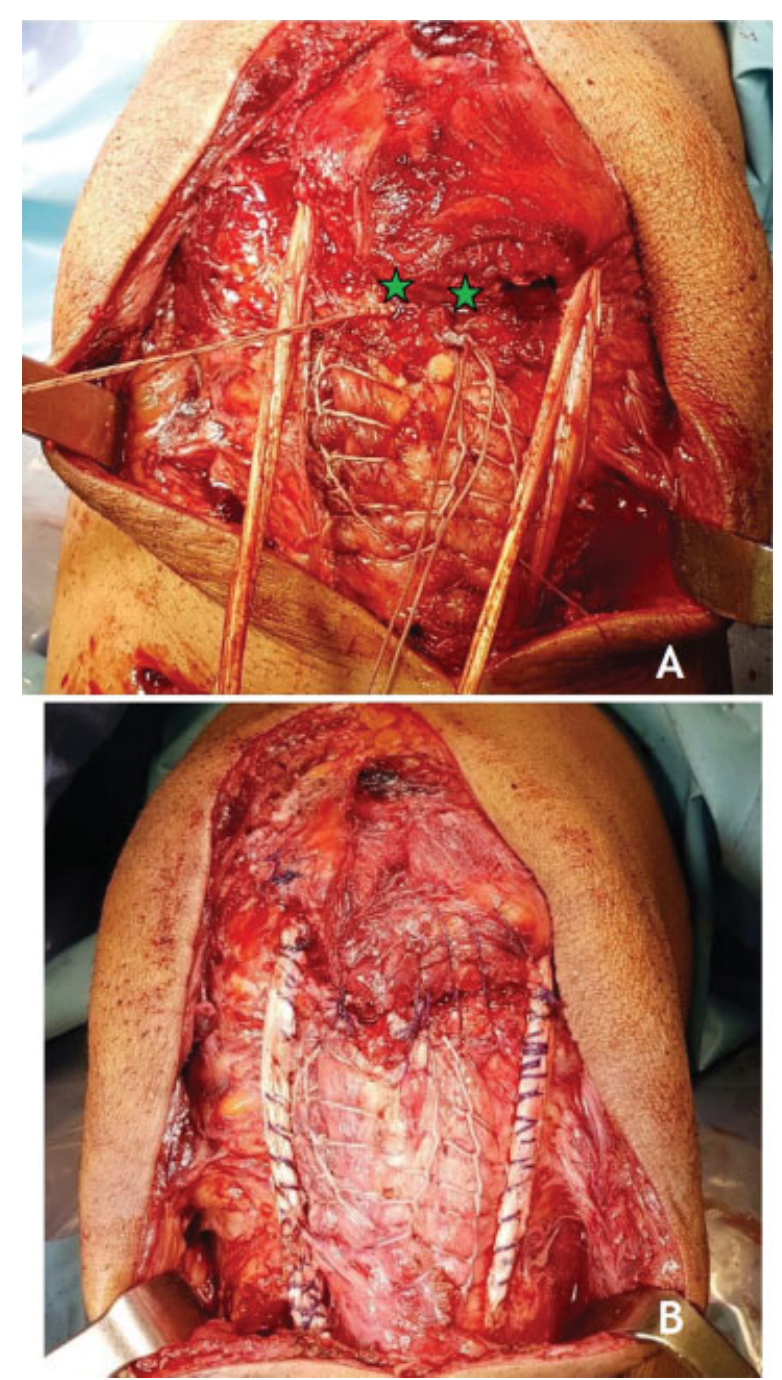

Fig. 5 Proceso final de reconstrucción. (A) Primero se anudan las anclas (estrellas verdes) en la extensión de la rodilla para lograr una buena coaptación de la interfaz tendón patelar-hueso. (B) En $30^{\circ}$ de flexión de la rodilla, se procede a tensar los injertos de forma simétrica a cada lado de la rótula y tras comprobar que el tracking en flexo extensión es adecuado, se suturan entre sí de forma latero-lateral. Se flecta la rodilla en $90^{\circ}$ para instalar un tornillo bioabsorbible de 6 mm de diámetro en el túnel tibial.

\section{Discusión}

La reconstrucción del tendón patelar con el uso de autoinjertos ipsilaterales de STG es segura, efectiva y validada desde el punto de vista clínico y funcional. ${ }^{9,25-27}$ Además, es un constructo de resistencia probada ${ }^{34,35}$ (la configuración en doble banda aporta una resistencia máxima a la carga de $2330 \mathrm{~N}$ ), que asegura una integración adecuada de los injertos. ${ }^{28}$ Estos últimos, al ser cosechados rutinariamente en la reconstrucción del ligamento cruzado anterior, permiten realizar una cirugía técnicamente reproducible, pese a la infrecuencia de esta patología.

Existen estudios de seguimiento a mediano plazo que respaldan la técnica descrita, ${ }^{25}$ con buenos resultados respecto a la movilización precoz, recuperación funcional y retorno a actividades de la vida diaria, con mínimas 
complicaciones locales. Pese a esas ventajas, es importante mencionar que generalmente los pacientes persisten con hipotrofia cuadricipital comparativa y no logran el mismo nivel de retorno a actividades deportivas prelesionales, siendo el momento de la reconstrucción el factor que parece tener mayor correlación con los resultados clínicos.

Ecker y col $^{36}$ describe por primera vez este procedimiento en 1979 y junto a otros autores posteriores ${ }^{37}$ refuerzan esta reconstrucción con cerclaje de alambre entre la rótula y la TAT, justificándolo como una medida de protección para la movilización articular precoz y para la liberación de la tensión de las partes blandas. Shelbourne y $\operatorname{col}^{38}$ reporta un refuerzo con cable de acero Dall-Miles. Sin embargo, todos esos dispositivos pueden limitar el rango de flexión de la rodilla y requieren a corto plazo una segunda intervención para su retiro. Casey y $\mathrm{col}^{37}$ recomienda su remoción tras 6 meses de evolución postoperatoria, con el fin de permitir a los tejidos alcanzar una resistencia suficiente. Maffuli y $\mathrm{col}^{25}$ descartó el riesgo de fallas en la reconstrucción sin su uso, lo que permite realizar el procedimiento en un sólo tiempo quirúrgico.

Debido a su baja incidencia, la mayor evidencia existente respecto al manejo de estas lesiones se basa en reportes de casos aislados y de forma minoritaria en estudios de series de casos no controlados.

Por el momento, no existen estudios comparativos que evalúen las diferentes técnicas de reconstrucción, por lo que no existe consenso en un gold-standard para el tratamiento de estas lesiones.

\section{Nuestro Enfrentamiento}

El diagnóstico de estas lesiones es clínico, por lo que una adecuada anamnesis y examen físico, asociado a una radiografía comparativa bilateral de rodilla que evidencie alteración en la altura patelar del lado afectado, son confirmatorios.

Usualmente los pacientes se presentan con déficit de extensión activa de la rodilla, depresión cutánea local, gap infrapatelar y atrofia cuadricipital ipsilateral. Debido al proceso inflamatorio y hemartrosis, en algunos casos estas lesiones pueden pasar desapercibidas en la urgencia ante un examinador no entrenado, generando retraso en el diagnóstico y roturas crónicas. ${ }^{39}$ En ese escenario, es de gran utilidad la resonancia nuclear magnética, que no solo evalúa la ubicación de la lesión, sino que también descarta alteraciones articulares asociadas. El uso de la ecografía es cuestionable por ser operador dependiente y en la práctica no logra ser un examen confirmatorio.

Ante ese tipo de diagnóstico, es fundamental descartar patologías médicas de base no pesquisadas previamente, que generen debilidad de la interfaz hueso tendón y tratarlas de forma eficiente, pues de lo contrario persistirá el riesgo de reintervenciones.

El hiperparatoroidismo secundario y terciario a consecuencia de la insuficiencia renal crónica, es una causa reconocida de lesiones atípicas del aparato extensor en pacientes jóvenes, ${ }^{40-43}$ tales como: rotura tendínea bilateral, rotura asincrónica del tendón contralateral o re-roturas debido, principalmente, a la resorción ósea subperióstica que ésta produce. Por su parte, los trastornos vasculares endoteliales en patología reumatológica, sumado al consumo de corticoides, provocan alteraciones estructurales del colágeno y microtrombosis que afectan la oxigenación tisular local, poniendo en riesgo la reparación primaria del tendón. ${ }^{39}$

Nos parece una opción adecuada el uso de anclas como procedimiento combinado de refuerzo al aumento con autoinjerto STG. Así evitamos realizar retunelización ósea vertical a nivel patelar, evitando perdida de stock óseo, convergencia con el túnel horizontal (necesario en la reconstrucción) y aumento del riesgo de fractura, con resultados descritos clínicamente como similares a la técnica de suturas transpatelares, sin embargo de mayor costo $^{44-46}$ (-Fig. 6).

El túnel tibial tiene su justificación en la distribución simétrica de la fuerza entre ambos autoinjertos, evitando alteraciones en el tracking patelar. El uso de tornillo bioabsorbible a nivel tibial evita un efecto limpiaparabrisas del injerto semitendinoso dentro del túnel que dificulte su osteointegración. A nivel rotuliano, el túnel óseo de 4,5mm es lo suficientemente pequeño para contener de forma ajustada el paso de ambos autoinjertos sin necesidad de tornillo bioabsorbible adicional.

Debido a la buena experiencia con el uso del ácido tranexámico endovenoso en el control del sangrado intraoperatorio en dosis de $10 \mathrm{mg} / \mathrm{kg}$, no utilizamos isquemia local para realizar el procedimiento. ${ }^{47,48}$ Eso genera una ventaja adicional, que es la correcta evaluación de la altura patelar al no generarse un efecto tenodésico cuadricipital por tracción muscular a consecuencia de la activación del torniquete.

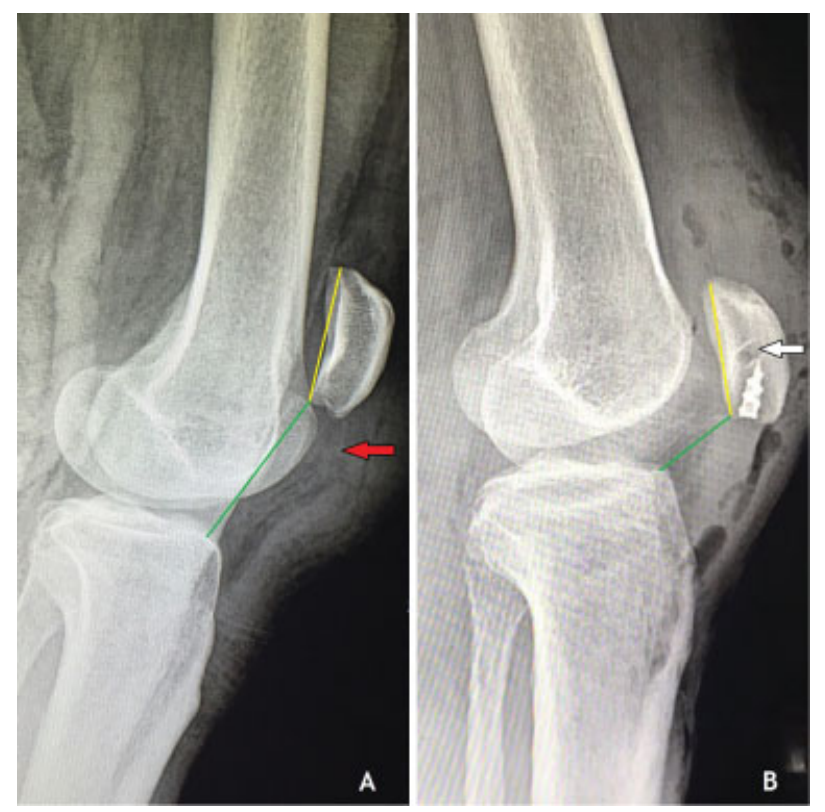

Fig. 6 Radiografías laterales de rodilla comparativas en paciente con rotura recidivante del tendón patelar. (A) Preoperatorio. Nótese patela alta según índice Caton-Deschamps (líneas de colores). Se aprecia imagen radiolúcida que demarca el trayecto del tendón patelar, interrumpido en interfaz hueso-tendón proximal (flecha roja). (B) Postoperatorio. Nótese en rótula las anclas normoposicionadas y sobre ésas, el túnel rotuliano (flecha blanca). Normalización del índice Caton-Deschamps. 
64 Técnica de reconstrucción en roturas recidivantes del tendón patelar Díaz Allende y col.

Responsabilidades Éticas de la Publicación

\section{Protección de personas y animales}

Para esta investigación no se han realizado experimentos en seres humanos ni en animales. En este artículo se realizó el consentimiento informado de investigación aprobado por el Comité de Ética a los pacientes que permitieron la presentación de sus fotografías y estudio imagenológico, preservando la confidencialidad de los datos.

\section{Declaración y verificación de la presentación}

Este trabajo no ha sido previamente publicado ni presentado simultáneamente en otra revista y tiene la autorización de los autores y su establecimiento asistencial.

\section{Conflicto de Intereses}

La realización de este estudio no presenta conflicto de intereses por parte de los autores.

\section{Agradecimientos}

Al Dr. David Figueroa P. de la Clínica Alemana de Santiago, por su gentil revisión del manuscrito original. A los Doctores Carlos Bolomey E. y Javier Cayazaya D. del Hospital Clínico FUSAT de Rancagua, por otorgarle al autor sus perspectivas en el manejo de estas lesiones.

A todo el equipo de Traumatología y Kinesiología del Hospital Ricardo Valenzuela Sáez de Rengo por el apoyo mostrado en la realización de este artículo.

\section{Bibliografía}

1 Clayton RA, Court-Brown CM. The epidemiology of musculoskeletal tendinous and ligamentous injuries. Injury 2008;39(12):1338-1344

2 Saragaglia D, Pison A, Rubens-Duval B. Acute and old ruptures of the extenso rapparatus of the knee in adults (excluding knee replacement). Orthop Traumatol Surg Res 2013;99:67-76

3 Negrin LL, Nemecek E, Hajdu S. Extensor mechanism ruptures of the knee: differences in demographic data and long-term outcome after surgical treatment. Injury 2015;46(10):1957-1963

4 Figueroa D, Figueroa F, Calvo R. Patellar Tendinopathy: Diagnosis and Treatment. J Am Acad Orthop Surg 2016;24(12):e184-e192

5 Blazina ME, Kerlan RK, Jobe FW, Carter VS, Carlson GJ. Jumper's knee. Orthop Clin North Am 1973;4(03):665-678

6 Kelly DW, Carter VS, Jobe FW, Kerlan RK. Patellar and quadriceps tendon ruptures-jumper's knee. Am J Sports Med 1984;12(05): 375-380

7 Kannus P, Józsa L. Histopathological changes preceding spontaneous rupture of a tendon. A controlled study of 891 patients. J Bone Joint Surg Am 1991;73(10):1507-1525

8 Ramseier LE, Werner CML, Heinzelmann M. Quadriceps and patellar tendon rupture. Injury 2006;37(06):516-519

9 Gilmore JH, Clayton-Smith ZJ, Aguilar M, Pneumaticos SG, Giannoudis PV. Reconstruction techniques and clinical results of patellar tendon ruptures: Evidence today. Knee 2015;22(03):148-155

10 Garner MR, Gausden E, Berkes MB, Nguyen JT, Lorich DG. Extensor Mechanism Injuries of the Knee: Demographic Characteristics and Comorbidities from a Review of 726 Patient Records. J Bone Joint Surg Am 2015;97(19):1592-1596

11 Cadambi A, Engh GA. Use of a semitendinosus tendon autogenous graft for rupture of the patellar ligament after total knee arthroplasty. A report of seven cases. J Bone Joint Surg Am 1992;74(07):974-979

12 Bek D, Demiralp B, Kömürcü M, Sehirlioğlu A. Neglected patellar tendon rupture: a case of reconstruction without quadriceps lengthening. J Orthop Traumatol 2008;9(01):39-42

13 Van der Bracht H, Verdonk R, Stuyts B. Augmentation of a patellar tendon repair with an autologous semitendinosus graft. Acta Orthop Belg 2009;75(03):417-419

14 Milankov MZ, Miljkovic N, Stankovic M. Reconstruction of chronic patellar tendon rupture with contralateral BTB autograft: a case report. Knee Surg Sports Traumatol Arthrosc 2007;15(12):1445-1448

15 Temponi EF, Camelo N, Tuteja S, et al. Reconstruction of chronic patellar tendon rupture with contralateral bone-tendon-bone autograft. Knee Surg Sports Traumatol Arthrosc 2017;25(08): 2468-2473

16 ElGuindy A, Lustig S, Servien E, et al. Treatment of chronic disruption of the patellar tendon in Osteogenesis Imperfecta with allograft reconstruction. Knee 2011;18(02):121-124

17 Crossett LS, Sinha RK, Sechriest VF, Rubash HE. Reconstruction of a ruptured patellar tendon with achilles tendon allograft following total knee arthroplasty. J Bone Joint Surg Am 2002; 84-A(08):1354-1361

18 Lewis PB, Rue JP, Bach BR Jr. Chronic patellar tendon rupture: surgical reconstruction technique using 2 Achilles tendon allografts. J Knee Surg 2008;21(02):130-135

19 Labib SA, Wilczynski MC, Sweitzer BA. Two-layer repair of a chronic patellar tendon rupture: a novel technique and literature review. Am J Orthop 2010;39(06):277-282

20 Levin PD. Reconstruction of the patellar tendon using a dacron graft: a case report. Clin Orthop Relat Res 1976;(118):70-72

21 Fukuta S, Kuge A, Nakamura M. Use of the Leeds-Keio prosthetic ligament for repair of patellar tendon rupture after total knee arthroplasty. Knee 2003;10(02):127-130

22 Naguib AM, Sefton GK. Recurrent bilateral rupture of the patellar tendons: Tendon replacement using polyester connective tissue prosthesis. Injury 2006;37:379-382

23 Naim S, Gougoulias N, Griffiths D. Patellar tendon reconstruction using LARS ligament: surgical technique and case report. Strateg Trauma Limb Reconstr 2011;6(01):39-41

24 Mihalko WM, Vance M, Fineberg MJ. Patellar tendon repair with hamstring autograft: a cadaveric analysis. Clin Biomech (Bristol, Avon) 2010;25(04):348-351

25 Maffulli N, Del Buono A, Loppini M, Denaro V. Ipsilateral hamstring tendon graft reconstruction for chronic patellar tendon ruptures: average 5.8-year follow-up. J Bone Joint Surg Am 2013;95(17):e1231-e1236

26 Sundararajan SR, Srikanth KP, Rajasekaran S. Neglected patellar tendon ruptures: a simple modified reconstruction using hamstrings tendon graft. Int Orthop 2013;37(11): 2159-2164

27 Spoliti M, Giai Via A, Padulo J, Oliva F, Del Buono A, Maffulli N. Surgical repair of chronic patellar tendon rupture in total knee replacement with ipsilateral hamstring tendons. Knee Surg Sports Traumatol Arthrosc 2016;24(10):3183-3190

28 Chen B, Li R, Zhang S. Reconstruction and restoration of neglected ruptured patellar tendon using semitendinosus and gracilis tendons with preserved distal insertions: two case reports. Knee 2012;19(04):508-512

29 Van der Zwal P, Van Arkel ERA. Recurrent patellar tendon rupture: reconstruction using ipsilateral gracilis and semitendinosus tendon autografts. Injury 2007;38:320-323

30 Järvelä T, Halonen P, Järvelä K, Moilanen T. Reconstruction of ruptured patellar tendon after total knee arthroplasty: a case report and a description of an alternative fixation method. Knee 2005;12(02):139-143

31 Krackow KA, Thomas SC, Jones LC. A new stitch for ligament-tendon fixation. Brief note. J Bone Joint Surg Am 1986;68(05):764-766 
32 Capiola D, Re L. Repair of patellar tendon rupture with suture anchors. Arthroscopy 2007;23(08):906.e1-906.e4

33 Insall J, Salvati E. Patella position in the normal knee joint. Radiology 1971;101(01):101-104

34 Hamner DL, Brown CH Jr, Steiner ME, Hecker AT, Hayes WC. Hamstring tendon grafts for reconstruction of the anterior cruciate ligament: biomechanical evaluation of the use of multiple strands and tensioning techniques. J Bone Joint Surg Am 1999;81(04): 549-557

35 Tashiro T, Kurosawa H, Kawakami A, Hikita A, Fukui N. Influence of medial hamstring tendon harvest on knee flexor strength after anterior cruciate ligament reconstruction. A detailed evaluation with comparison of single- and double-tendon harvest. Am J Sports Med 2003;31(04):522-529

36 Ecker ML, Lotke PA, Glazer RM. Late reconstruction of the patellar tendon. J Bone Joint Surg Am 1979;61(6A):884-886

37 Casey MT Jr, Tietjens BR. Neglected ruptures of the patellar tendon. A case series of four patients. Am J Sports Med 2001;29 (04):457-460

38 Shelbourne KD, Darmelio MP, Klootwyk TE. Patellar tendon rupture repair using Dall-Miles cable. Am J Knee Surg 2001;14(01):17-20, discussion 20-21

39 Razzano CD, Wilde AH, Phalen GS. Bilateral rupture of the infrapatellar tendon in rheumatoid arthritis. Clin Orthop Relat Res 1973;(91):158-161

40 Shiota E, Tsuchiya K, Yamaoka K, Kawano O. Spontaneous major tendon ruptures in patients receiving long-term hemodialysis. Clin Orthop Relat Res 2002;(394):236-242
41 Chen CM, Chu P, Huang GS, Wang SJ, Wu SS. Spontaneous rupture of the patellar and contralateral quadriceps tendons associated with secondary hyperparathyroidism in a patient receiving longterm dialysis. J Formos Med Assoc 2006;105(11):941-945

42 Grecomoro G, Camarda L, Martorana U. Simultaneous chronic rupture of quadriceps tendon and contra-lateral patellar tendon in a patient affected by tertiary hyperparatiroidism. J Orthop Traumatol 2008;9(03):159-162

43 Seng C, Lim YJ, Pang HN. Spontaneous disruption of the bilateral knee extensor mechanism: a report of two cases. J Orthop Surg (Hong Kong) 2015;23(02):262-266

44 Bushnell BD, Byram IR, Weinhold PS, Creighton RA. The use of suture anchors in repair of the ruptured patellar tendon: a biomechanical study. Am J Sports Med 2006;34(09):1492-1499

45 Ettinger M, Dratzidis A, Hurschler C, et al. Biomechanical properties of suture anchor repair compared with transosseous sutures in patellar tendon ruptures: a cadaveric study. Am J Sports Med 2013;41(11):2540-2544

46 Lanzi JT Jr, Felix J, Tucker CJ, et al. Comparison of the Suture Anchor and Transosseous Techniques for Patellar Tendon Repair: A Biomechanical Study. Am J Sports Med 2016;44(08): 2076-2080

47 Melvin JS, Stryker LS, Sierra RJ. Tranexamic Acid in Hip and Knee Arthroplasty. J Am Acad Orthop Surg 2015;23(12):732-740

48 Xie J, Ma J, Yao H, Yue C, Pei F. Multiple Boluses of Intravenous Tranexamic Acid to Reduce Hidden Blood Loss After Primary Total Knee Arthroplasty Without Tourniquet: A Randomized Clinical Trial. J Arthroplasty 2016;31(11):2458-2464 